\title{
Excitons in incompressible quantum liquids
}

\section{Citation}

Rashba, E. I. 1995. Excitons in Incompressible Quantum Liquids. Pure and Applied Chemistry 67, no. 3. doi:10.1351/pac199567030409.

\section{Published Version}

doi:10.1351/pac199567030409

\section{Permanent link}

http://nrs.harvard.edu/urn-3:HUL.InstRepos:29407533

\section{Terms of Use}

This article was downloaded from Harvard University's DASH repository, and is made available under the terms and conditions applicable to Other Posted Material, as set forth at http:// nrs.harvard.edu/urn-3:HUL.InstRepos:dash.current.terms-of-use\#LAA

\section{Share Your Story}

The Harvard community has made this article openly available.

Please share how this access benefits you. Submit a story.

\section{Accessibility}




\title{
Excitons in incompressible quantum liquids
}

\author{
E. I. Rashba
}

Department of Physics, University of Utah, Salt Lake City 84112, USA and Landau Institute for Theoretical Physics, Moscow 117940, Russia

\begin{abstract}
A theory of excitons in semiconductors in the Fractional Quantum Hall (FQH) Effect regime is presented. Non-conventional properties of magnetoexcitons in this regime originate from the fact that elementary excitations of the FQH phases carry fractional charges. Properties of excitons strongly depend on the separation $h$ between electron and hole confinement planes. When this separation is not too small, $h \gtrsim l$, where $l$ is the magnetic length, excitons look like quasiatoms consisting of a valence hole and several fractional charges. Charge fractionalization results in a multiple-branch structure of the exciton energy spectrum. Symmetry classification of the branches is proposed, and their relation to the low-energy part of the Hilbert space of the charged elementary excitations of the FQH phases is established. Spectroscopic implications of the spectra classification, including the selection rules, are discussed.
\end{abstract}

\section{Introduction}

Excitons have been discovered and investigated in different inorganic and organic compounds during the last half-century. They show very different properties, have different size, binding energy, spin, coupling to phonons, etc. However, there is one basic property which is common for all of them: an exciton is a neutral particle consisting of a single electron and single hole, each carrying a charge equal to the elementary charge $e$ (A specific type of excitons in which both an electron and hole reside on different intramolecular orbitals of the same molecule we call molecular, or Frenkel, excitons). This common property of excitons is based on the fundamental law of the integer quantization of the electrical charge known for a century since the discovery of an electron.

More recently, in 1982-1983, a discovery has been made in the physics of semiconductors which changed fundamentally the established concepts about charged elementary excitations in solids. It was shown that under some conditions charged elementary excitations of twodimensional (2D) electronic phases carry fractional charges $e_{q}= \pm e / q$, where $q$ is an odd integer. These new elementary excitations, quasielectrons and quasiholes, can manifest themselves in different phenomena. In particular, they can participate in the formation of an absolutely new type of exciton. Such an exciton is a neutral entity consisting of a positive elementary charge $(+e)$, the usual valence hole, and $q$ quasielectrons, each of them carrying a fractional charge $(-e / q)$. For reasons which will become clear from what follows, we shall term them anyon excitons. Such an entity resembles an atom, since it consists of a "large" central charge and numerous fractional charges around it. One may expect that the spectroscopy of these 2D quasiatoms will possess simultaneously the properties which are specific for both atomic spectroscopy and the spectroscopy of conventional excitons. I wish to show in this paper that the distinctive feature of anyon excitons is a multiple-branch 
structure of their energy spectrum caused by the internal degrees of freedom of their anyon shells. The experimental spectroscopy of anyon excitons does not exist for the time being. However, our theoretical data strongly suggest that the multiple-branch spectrum of these excitons should be accessible for observation and investigation.

\section{Fractional Quantum Hall Effect and Fractional Electrical Charges}

Charge carriers acquire the exotic properties mentioned above when they appear against a background of 2D electronic phases called Incompressible Quantum Liquids (IQL). Electrons show $2 \mathrm{D}$ behavior and form $2 \mathrm{D}$ phases when they are confined in semiconductor heterojunctions and quantum wells having the width about $100 \AA$ or less. Their motion in the $z$-direction, i.e., perpendicular to the confinement plane, being strongly quantized, they retain only the lateral degrees of freedom. A strong magnetic field $H$ applied in the $z$-direction quenches the free motion of electrons in the $x, y$-plane. Their spectrum becomes completely discrete, it is determined by the Landau quantization $\varepsilon_{N}=\hbar \omega_{c}(N+1 / 2)$, where $\omega_{c}=e H / m c$ is a cyclotron frequency. The number of quantum states per unit area equals $n_{1}=1 / 2 \pi l^{2}$ for each Landau level, where $l=(c \hbar / e H)^{1 / 2}$ is the magnetic length. If $H$ is sufficiently strong, and $T=0$, then:

i) The filling factor $\nu=n / n_{1}$ is less than one, $\nu<1$, since electrons populate only the lowest Landau level ( $\mathrm{N}=0, n$ is the electron concentration), and

ii) the characteristic Coulomb energy $\varepsilon_{C}=e^{2} / \epsilon l$ is less than $\hbar \omega_{c}, \varepsilon_{C} \ll \hbar \omega_{c}$, where $\epsilon$ is a dielectric constant. Hence the Landau level mixing may be neglected.

Under these conditions $\varepsilon_{C}$ is the only quantity of the dimensionality of energy which enters the theory. There are no other competing quantities. Therefore, the properties of the ground state are determined completely by the filling factor $\nu$, and $\varepsilon_{C}$ determines the energy scale of elementary excitations.

Now we are in position to formulate the fact which is of a special importance. When $\nu$ takes the values $\nu_{p / q}=p / q$, where $p$ is an integer, and $q$ is odd, the electron system condenses into an incompressible phase, the IQL. These liquids have unique properties which manifest themselves in the Fractional Quantum Hall Effect (FQHE)(1), a non-dissipative electron magnetotransport $\left(\sigma_{x x}=0\right)$ with quantized values of the Hall conductivity $\sigma_{x y}=$ $\left(e^{2} / 2 \pi \hbar\right) \nu_{p / q}$.

The notion of IQL's was introduced in the seminal paper by Laughlin (2), who established the basic properties of these liquids. They are as follows:

i) IQL's are homogeneous phases with the quantized densities $\nu=p / q$, where $p$ is an integer, and $q \neq 1$ is odd.

ii) Charged elementary excitations of IQL's, quasielectrons (QE) and quasiholes, have fractional charges $e_{q}= \pm e / q$.

iii) There are no "soft" branches of neutral elementary excitations in IQL's. The energy gap $\Delta$ for formation of a quasielectron-quasihole pair algebraically has the scale $\varepsilon_{C}$. However, it is numerically small as compared to $\varepsilon_{C}$, e.g., for the most stable $\nu=1 / 3$ - IQL it equals $\Delta \approx 0.1 \varepsilon_{C}$.

Exotic properties of the charge carriers are not restricted by the charge fractionalization. Halperin has shown that they also obey a fractional statistics, which is intermediate between the Bose and Fermi statistics. Such particles are termed anyons. The statistical properties of these quasiparticles may be expressed either in terms of wave functions (3), or in terms of the filling of the phase space (4).

The lowest branch of the spectrum of neutral elementary excitations is termed a magnetoroton branch $\omega_{M R}(k)$, where $k$ is the quasimomentum (Girvin et al., Ref. 5). The magnetoroton may be modeled either as a charge density wave (5), or as a quasiexciton consisting of a bound quasielectron-quasihole pair (6). Energy of large- $k$ magnetorotons is 
related to the gap, $\hbar \omega_{M R}(k \rightarrow \infty)=\Delta$. There is a continuum above the magnetoroton branch whose states are not classified yet.

\section{Spectroscopy of IQL's: Excitons}

IQL's were discovered by means of magnetotransport experiments which are a splendid tool for investigating the ground state properties. However, optical spectroscopy seems to be an indispensable tool for investigating the spectrum of elementary excitations. To be effective in resolving tiny features related to the properties of IQL's, spectroscopy should have a resolution much better than $\Delta$, which is about $1 \mathrm{meV}$ for magnetic fields about $10 \mathrm{~T}$.

There are successful observations of the specific features in the spectra of the intrinsic emission from IQL's in the vicinity of fractional fillings $\nu_{p / q}$ which correlate with magnetotransport data (7). Resonant Raman scattering permitted the measurement of the frequency of the $k=0$ neutral excitation (8). Highly pronounced features were observed in the extrinsic emission spectra coming from the trapping of the electrons from a confinement layer by neutral impurities (9). They were successfully treated in terms of cusp-strengths (10) and used for measuring $\Delta$ values for numerous IQL's (11).

After the signatures in optical spectra of the formation of IQL's have been observed, the main problem shifts from taking the spectra to the assignment and treatment of them. There are electronic correlations which determine the basic properties of IQL's. This means that the traditional methods and concepts of the spectroscopy of semiconductors which are based either on neglecting the electron-electron interaction, or on taking it into account in the self-consistent approximation, are inapplicable to IQL's. Assignment of experimental data should be based on a theory which takes into account the basic properties of IQL's in a proper way.

It is clear from the basic principles that the intrinsic recombination spectra of IQL's are dominated by excitons. Indeed, in a strong magnetic field the binding energy of an exciton increases from about $m e^{4} / \epsilon^{2} \hbar^{2}$ up to $e^{2} / \epsilon l$. Their ratio is just the small parameter of the theory $\varepsilon_{C} / \hbar \omega_{c} \ll 1$. The main problem is: how does an IQL change the properties of excitons?

\section{Theoretical Concepts. Governing parameter}

In our model exciton consists of a hole and a screening electric charge induced in the electron confinement layer where the IQL forms. A hole lives in a different confinement layer which is parallel to the electron confinement layer and is separated from it by a distance $h$. In an ideal scheme the confinement layers have a zero width, i.e., they are confinement planes. In real systems their widths are of the order of $100 \AA$. A $2 \mathrm{D}$ quasimomentum can be always ascribed to an exciton even in a magnetic field since it is a neutral entity. Different quantum numbers depend on the specific internal structure of the exciton, see below.

Depending on the ratio $h / l$ there are two limiting cases.

a. $h / l \ll 1$. In this case the perturbing field of a hole is very strong since $\varepsilon_{C} \gg \Delta$, and the electron density in the vicinity of a hole strongly deviates from $\nu n_{1}$. This limit is most interesting from the point of view of a strong renormalization of the exciton properties, especially its dispersion law, and the existence of a hidden symmetry trivializing its optical spectrum.

The energy spectrum can not be found analytically. The most powerful tools are exact numerical calculations for a fw particle systems; actually, the number of particles $N \leq 10$. A spherical geometry (12) in which a magnetic monopole is placed in the center of a sphere is especially convenient. The magnetic flux through the sphere, $2 S \phi_{0}$, should be a multiple of the flux quantum $\phi_{0}=2 \pi \hbar c / e$. The radius of the sphere $R=S^{1 / 2} l$. The relation between the angular momentum of a quantum state on the sphere, $L$, and the quasimomentum of the corresponding state on the plane, $k$, is usually established by the prescription $L=R k$. 
The spherical geometry is most appropriate for spectroscopic applications since it retains a continuum rotation group inherent in the real plane geometry and excludes edge effects.

b. $h / l \gg 1$. In this case the perturbation produced by a hole is small as compared to $\Delta$, and the size of the exciton $(\approx h)$ is large as compared to a size of an anyon $(\approx l)$. Under these conditions an exciton is an atom-like entity having internal anyon degrees of freedom. Its energy spectrum should comprise a multiplicity of branches. There are appropriate analytical approaches to the problem. This limit permits one to bridge the exciton spectroscopy of IQL's and the main body of the low-energy physics of the FQHE, and opens a new approach to the latter. Actually, excitons are few-anyon systems where the interaction and dynamics of anyons may be investigated theoretically and controlled by optical experiments.

I will show in this paper that the events in the intermediate region $h \approx l$ where the multiple-branch exciton spectrum emerges are extremely instructive in disclosing the deep connections existing between the physics of excitons and the physics of many-anyon systems. A hole works as a probe which explores the quantum states of interacting anyons. When $h$ changes, the exciton energy spectrum changes strongly. Different branches of it intersect and mutually transform. The separation $h$ acquires the role of a governing parameter controlling the exciton spectrum (13).

\section{Small $h / l$ values. Dressing of an Exciton by IQL}

When $h$ is small, $h / l<1 / 2$, the energy spectrum of a system comprising a single exciton and an $\nu=1 / 3$ - IQL consists of a single exciton branch and a structureless continuum above it, Fig. 1a. Coupling of the exciton to the IQL strongly perturbs the liquid around it and changes the exciton properties. Nevertheless, exciton remains a stable particle with a well defined spectrum; for more detail see Ref. 14. Exciton dispersion is strongly suppressed as compared to a bare exciton, i.e., an exciton in an empty crystal. An increase in the exciton effective mass may be considered as a polaron effect caused by the dressing of it by neutral elementary excitations of the IQL. As distinct from the conventional polaron effect, the electron entering the exciton belongs to the same Fermi sea which dresses the exciton.
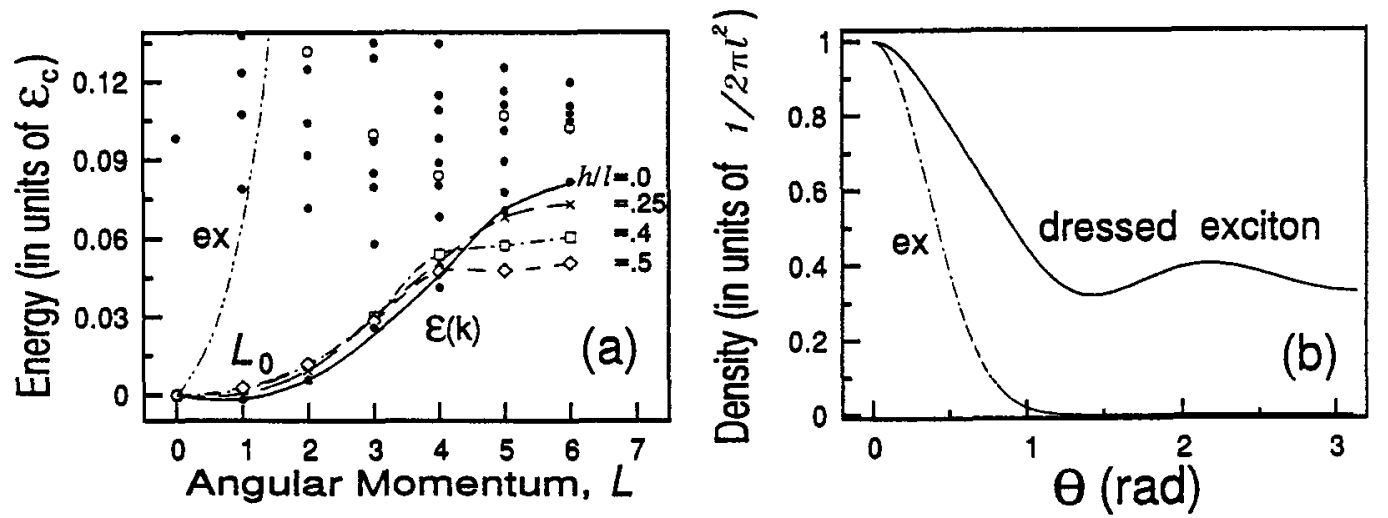

Fig. 1. (a) Lower part of the energy spectrum of an exciton against a background of an IQL consisting of $N_{e}=6$ electrons in a spherical geometry. For $h=0$ the spectrum of a bare exciton (ex), a dressed exciton, $\varepsilon(k)$, and the "continuum" above it are shown. Only $\varepsilon(k)$ is shown for three different values of $h$. The energy $\varepsilon(k=0)$ is chosen as an origin for all curves. Multiplicative states are shown by open dots; they also display the magnetoroton dispersion law (From Ref. 15).

(b) Distribution of the electron density around a hole in the $k=0$ state for a bare exciton (ex) and a dressed exciton for $h=0$. For large values of $\Theta \approx \pi$ electron density is close to the density $3 / 8$ of the $\nu=1 / 3-$ IQL in the finite system. 
Electron density in the center of a $k=0$ bare exciton equals $n_{1}$ which is the maximum density compatible with the Pauli exclusion principle. For $h=0$ this value remains unchanged, hence, the distribution of the electron density becomes flatter to ensure the electronic charge conservation; this distribution is shown in Fig. 1b. For $h=0$ the electron-hole system possesses a hidden symmetry: frequencies of all allowed transitions coincide exactly with the transition frequency of a bare exciton. The states to (from) which transitions are allowed are termed multiplicative states. They constitute only a small part of all excited states. In Fig. 1a these states are shown by open dots. The lowest dot corresponds to a transition from the ground state of the system, and other open dots to transitions from single-magnetoroton states. Absence of the shift of the $k=0$ exciton level may be treated as an exact cancellation of the down-shift caused by polaron effect and the up-shift caused by the Pauli exclusion principle (since the IQL fills a considerable part of the electron phase space). For $h \neq 0$ the compensation is no more complete and the up-shift dominates.

It is seen from Fig. 1a that up to $h / l=0.5$ the continuum remains structureless, and the gap which forms between it and the exciton branch remains narrow.

6. Large $h / l$ values. Anyon excitons

In this limit an exciton may be considered as a bound state of a valence hole and $q$ QE's as was argued in Sect. 4. In a strong magnetic field every charged particle possesses a single degree of freedom and a single quantum number. Therefore, an exciton possesses $(q+1)$ quantum numbers. In a plane geometry two of them may be chosen as the 2D exciton quasimomentum $\mathbf{k}$, and $(q-1)$ describe internal degrees of freedom. Therefore, a conventional (electron-hole) magnetoexciton, $q=1$, has no internal quantum numbers. This is a well known fact.

A simple model of an anyon exciton includes two QE's (16); therefore, $q=2$, and there is a single internal degree of freedom. For $k=0$ the internal angular momentum $M$ may be chosen as the corresponding quantum number ( $M \leq 0$ since QE's carry negative charges). The wave functions of this system may be written in the representation of pseudo (envelope) functions (3):

$$
\left.\Psi_{\mathbf{k n}}\left(z_{1}, z_{2}, z_{3} \mid \alpha\right) \propto \exp \left\{i \mathbf{k R}+i\left(\rho_{x} Y-\rho_{y} X\right) / 2\right\} \exp \left\{-(1 / 4)(\vec{\rho}-\vec{\kappa})^{2}\right)\right\}|z|^{\alpha} \bar{z}^{n} \exp \left(-|z|^{2} / 16\right) .
$$

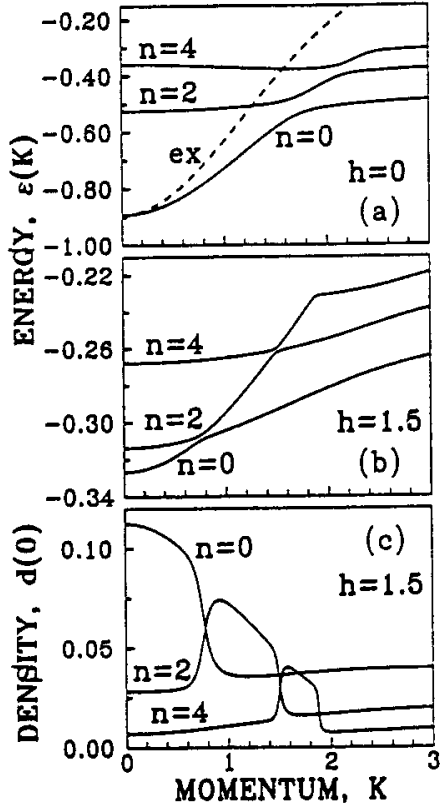

Fig. 2. (a), (b): Lower part of the energy spectrum of an anyon exciton consisting of a hole and two $(-e / 2)$ QE's. When $h$ increases, anticrossings become narrower, and $\varepsilon(k)$ for the ground exciton state becomes flatter. The dispersion law of an conventional magnetoexciton (ex) is shifted to facilitate a comparison with $n=0$ curve. $h$ in units of $l, \varepsilon(k)$ in units of $\varepsilon_{C}$. (c): Electron density at the hole in the three lower states (From Ref. 16). 
In this equation we use complex coordinates $z=x+i y$ for QE's $\left(z_{1}\right.$ and $\left.z_{2}\right)$ and a hole $\left(z_{3}\right)$, and also Jacobi coordinates $z=z_{1}-z_{2}$ and $z_{0}=\left(z_{1}+z_{2}\right) / 2$ for QE's. From $z_{0}$ and $z_{3}$ new coordinates are formed by the usual exciton transformation. Therefore, the final complex coordinates are $z, \zeta=z_{0}-z_{3}$, and $Z=\left(z_{0}+z_{3}\right) / 2$. The vectors $\mathbf{R}, \vec{\rho}$ and $\mathbf{r}$ correspond to complex coordinates $Z, \zeta$ and $z$, respectively; $\vec{\kappa}=\hat{z} \times \mathbf{K}, \hat{z}$ is a unit vector in the direction perpendicular to the confinement plane. The quantum number $n=|M|$. Most of the factors in this equation are completely determined by the magnetic symmetry. The only non-trivial factor is $|z|^{\alpha}$, it comes from the fractional statistics of QE's. For a two-anyon model the charge of QE's equals $e_{2}=-e / 2$, and $\alpha=-1 / 2$. For comparison, $\Psi_{\mathrm{kn} n}$ is even in $\bar{z}$ for bosons, and odd in $\bar{z}$ for fermions. At $k=0$ the Hamiltonian is diagonal in $n$, and the functions $\Psi_{\mathbf{k} n}$ permit us to calculate the energy levels explicitly. For $k \neq 0$ the anyon-hole interaction is non-diagonal in $n$, and the Hamiltonian must be diagonalized numerically. The results for the lower Landau levels are shown in Fig. 2. They were obtained with a Coulomb interaction between all particles.

The spectrum consists of multiple branches as shown in Fig. 2. Their positions strongly depend on $h / l$, so that with increasing $h / l$ the branches with large $n$ values move down. The transformation of the spectrum is accompanied by numerous intersections of the branches. All branches are flat as compared to the spectrum of a conventional magnetoexciton shown as the dashed line in Fig. 2a. This behavior is in agreement with Fig. 1a, but now it may be understood in somewhat different terms. When $k \rightarrow \infty$, one of the QE's moves away from the exciton. It seems obvious that a partial ionization of an exciton costs less energy than the full ionization of it. Therefore, the larger is $q$, the flatter the exciton spectrum is. The electron density in the point where the hole resides is shown in Fig. 2c for different quantum states. It is seen that every anticrossing of exciton branches is associated with a strong electron density exchange between them.

7. Intermediate $h / l$ values. Emergence of the multiple-branch spectra

Two absolutely different pictures of the exciton spectrum were obtained in Sects. 5 and 6 for two opposite limit cases. The question is: when and how do these pictures match?

"When" means: how large is the ratio $h / l$ when the transformation sets in? If it occurs at large values of $h / l$, the transition can not be investigated theoretically in the spherical geometry since $h$ can not be chosen larger than about $R / 2$; actually, $h / l \lesssim 1.5$. For too large values of $h$ the effect also can not be investigated experimentally since the optical transition probability is controlled by the overlap between electron and hole wave functions, so that it decreases exponentially with $h$. We show below that for $\nu=1 / 3$ and $2 / 5$ - IQL's the transformation sets in in a convenient region $h / l \approx 1$.

"How" means: what happens to the original $(h=0)$ exciton branch and the continuum seen in Fig. la when $h / l$ increases? Where do the new branches come from? We show that they split off of the bottom of the continuum (and many of them move down), while the original branch moves up and looses its importance with increasing $h / l$.

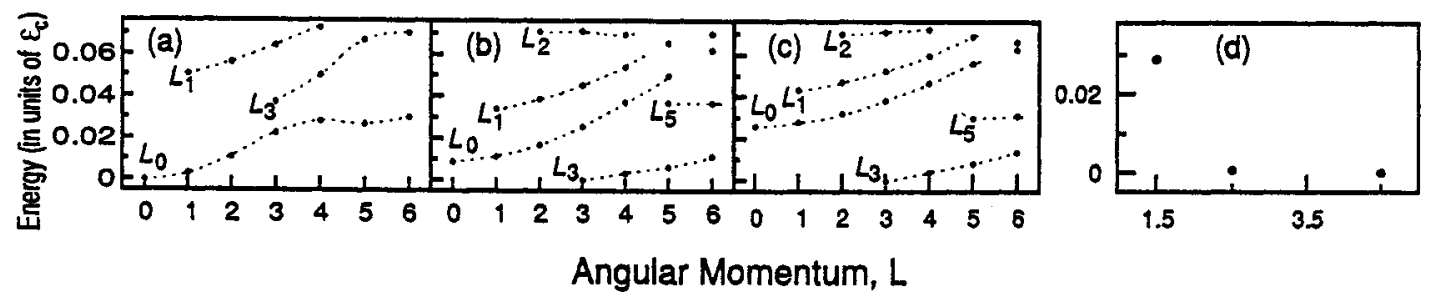

Fig. 3. Energy spectrum of the same system as in Fig. 1a for $h / l \geq 0.7$ : (a) $h / l=0.7$, (b) 1.0, and (c) 1.2. In (d) the energy spectrum of the $N_{e}=7$ system is shown (From Ref. 17). 
It is seen from Fig. 3 how the spectrum shown in Fig. 1a changes when $h / l$ increases. Already at $h / l=0.7$ two new branches starting at $L=3$ and $L=1$ emerge; they are designated as $L_{3}$ and $L_{1}$, respectively. The branch $L_{3}$ moves down with increasing $h / l$. The branch $L_{5}$ which appears later follows it. The origin of the branch $L_{6}$ is also seen in Fig. 3 c (at $L=6)$. Calculations performed for a larger system $\left(N_{e}=9, N_{h}=1\right)$ result in nearly the same data.

We are inaposition now to summarize the general regularities which follow from the calculations. New branches split off at the bottom of the continuum. The branches $L_{m}$ with $m \geq 3$ move down when $h / l$ increases and pass below the the original $L_{0}$ branch for $h / l \gtrsim 1$. The branches $L_{m}$ with $m=1,2$ remain above $L_{0}$. Therefore, the branch $L_{3}$ plays some special role. It plays the same role also for a $\nu=2 / 5-$ IQL.

\section{Exciton Branches and Hilbert Space of Quasielectrons}

An exciton existing against a background of an $\nu=1 / 3-$ IQL consists of a hole and three QE's. There exists a question: what are the values of the angular momentum in the lowenergy states of a composite consisting of these four particles? It can be shown from the general arguments based on the dynamics of the particles on a sphere and the limitations imposed by statistics, that for $\nu=1 / 3$ - IQL's the maximum angular momentum of three QE's equals

$$
\left(L_{Q E}\right)_{\max }=3 N_{e} / 2-6,
$$

and the angular momentum of a hole equals

$$
L_{h}=3 N_{e} / 2-3
$$

here $N_{e}$ is the full number of electrons on the sphere. The low-energy spectrum of three QE's in the absence of a hole may be found by calculations, for $N_{e}=7$ it is shown in Fig. $3 \mathrm{~d}$. It is seen that $\left(L_{Q E}\right)_{\max }=4.5$ in agreement with Eq. (1). It follows from Eqs. (1) and (2), and from the usual momenta addition rule that the angular momentum of an exciton built from a hole and three QE's can take the values

$$
L_{\mathrm{ex}} \geq L_{h}-\left(L_{Q E}\right)_{\max }=3 .
$$

This value does not depend on $N_{e}$ (15). Eq. (3) explains the special role the branch $L_{3}$ plays. Only $L_{m}$ branches with $m \geq 3$ may appear within the framework of the quasiparticle description, i.e., with an exciton as a composite built of a hole and low-energy QE's (cf. Sect. 6). Excitons with $m<3$ include highly excited states of the electron subsystem.

\section{Quasielectron-Hole Momentum Coupling Scheme}

The conclusion of Sect. 8 about the special role of the $L_{3}$ branch is based on general arguments. To understand the regularities which govern the appearance of different branches with $m \geq 3$, it is necessary to consider in more detail the low-energy spectrum of three QE's. The decomposition of the low-energy part of the three-QE Hilbert space of a seven-electron system into subspaces with different angular momenta is $4.5 \oplus 2.5 \oplus 1.5$, the two former states having the lower energy, Fig. 3d. Taking into account that $L_{h}=7.5$, and applying the momentum addition scheme one finds that the minimal angular momenta of excitons arising from the three QE subspaces are equal to 3, 5, and 6, respectively. These are just the minimum $L$ values for the branches $L_{3}, L_{5}$, and $L_{6}$ which appear in Fig. 3 . This observation implies the momentum coupling scheme for low-energy states which may be designated as $\{Q E\} h$ - coupling: the momentum $L_{Q E}$ which forms in the absence of a hole couples to $L_{h}$, and by addition of them the exciton angular momentum $L_{\mathrm{ex}}$ is formed. The 
$\{Q E\} h$ - coupling scheme was checked also for a larger $\nu=1 / 3$ system $\left(N_{e}=8, N_{h}=1\right)$, and for $\nu=2 / 5-$ IQL $\left(N_{e}=9, N_{h}=1\right)$; in the latter case an exciton includes five QE's. One can check by inspection that first emerge the excitons which originate from subspaces having lower energies, and between the excitons emerging from subspaces with comparable energies first come those which have lower values of $m$, i.e., the lesser exciton size.

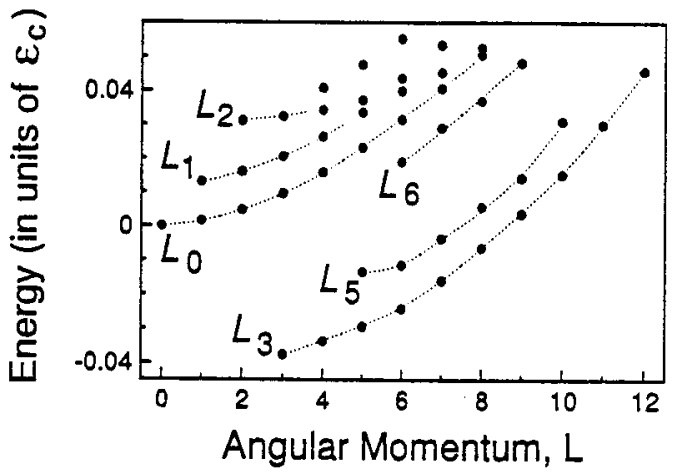

Fig. 4. Energy spectrum of the same system as in Fig. 1a for $h / l=1.4$ in a wide region of $L$ values. The number of states at the branches $L_{3}, L_{5}$, and $L_{6}$ is in agreement with the $\{Q E\} h-$ coupling scheme (From Ref. 17).

Exciton dispersion laws are shown in Fig. 3 only for $L \leq 6$ since the accuracy of the data decreases for larger momenta. However, for the systematics-of-states purposes it is instructive to consider the data in a larger range of $L$ values. They are shown in Fig. 4 and permit to check the $\{Q E\} h$ - coupling scheme. The number of states at the $L_{3}, L_{5}$, and $L_{6}$ branches coincides exactly with the requirements of the momentum addition rule, $L_{h}-L_{Q E} \leq L \leq L_{h}+L_{Q E}$. When $R \rightarrow \infty$, the branches which arise from the addition of the momenta turn into different branches of the exciton dispersion law in the plane geometry.

\section{Sphere-onto-Plane Projection. Spectroscopic Implications}

Real systems have a plane, and not a spheric geometry. Therefore, it is necessary to have a definite prescription for projecting the dispersion law of a composite particle between these two geometries. In the plane geometry the quasimomentum $\mathbf{k}$ is a quantum number; the energy depends on $k$, and the phase of $\mathbf{k}$ forms the space of degeneracy. In the spherical geometry the quantum numbers are $L$ and $L_{z}$; the energy depends on $L$, and $L_{z}$ forms the space of degeneracy. Therefore, there are the quantities $L$ and $k$ which should be connected by some relation. One more fact should be taken into account: for $L_{m}$ branches the values of $m$ do not depend on the system size. Therefore, $L_{m}$ is a quantum number, and it may be understood only as an internal angular momentum of a composite particle. The prescription based on these arguments is as follows: $L_{m}$ is the internal angular momentum, $L_{m}=|M|$, and $L-L_{m}=R k$. The latter equation generalizes the usual prescription $L=R k(12)$ as applied to composite particles.

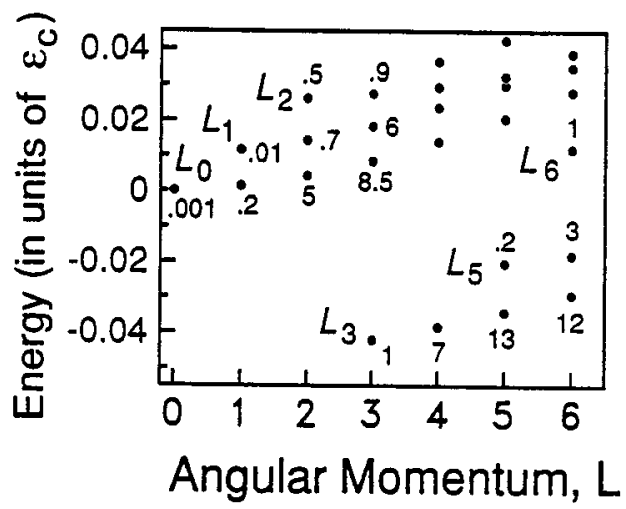

Fig. 5. Energy spectrum of the same system as in Fig. 1a for $h / l=1.5$. Numbers show calculated intensities of singlemagnetoroton transitions (in percents to the transition probability from multiplicative states, for $h=0$ ) (From Ref. 18). 
Identification of the left ends of the $L_{m}$ branches in the spherical geometry with the $k=0$ point in the plane geometry point implies some restrictions on the shape of $L_{m}$ curves, and also some selection rules for optical transitions. Since the dispersion law should show an extremum (usually a minimum) at $k=0$, one can expect that the slope of $L_{m}$ curves at their left end should tend to zero when $R \rightarrow \infty$. The slope of well developed curves $\left(L_{0}, L_{1}, L_{3}\right.$, and $L_{5}$ ) in Fig. 4 decreases with decreasing $L$.

In the $k=0$ point excitons may be classified according to the projection of the internal angular momentum $M$. Therefore, direct transitions to the ground state are allowed only from the $L_{0}$ branch. Magnetorotons also are composite particles. Therefore, they might be expected to have some internal momentum $M_{\mathrm{MR}}$ whose value is as yet unknown. Singlemagnetoroton transitions at the $k=0$ point are allowed from the exciton branches having $M=M_{\mathrm{MR}}$, and forbidden for all different branches. The transition probabilities found in the spherical geometry are expected to have i) a considerable magnitude at the left end and a weak $k$ dependence for allowed transitions, and ii) a small magnitude at the left end and a strong $k$ dependence for forbidden transitions. In Fig. 5 single-magnetoroton transition probabilities are shown for all points having an unambiguous assignment. The branch $L_{2}$ seems to be the only candidate for the allowed single-magnetoroton transition; the transition is weak, but its intensity shows a slow $L$ dependence. These data imply that magnetorotons are $L_{2}$ quasiparticles.

\section{Conclusion}

It was shown above that excitons possess unusual properties in the FQHE regime. These properties originate from the charge fractionalization, which manifests itself in the multiplebranch structure of the exciton energy spectra. The most favorable experimental procedure for observing exotic properties of excitons consists in changing the parameter $h / l$ under the conditions $\nu=$ const. The theory establishes a direct relation between the spectroscopy of excitons and the low-energy physics of the FQHE. The experimentally observable objects of the latter are hierarchical (many-particle) states and the gaps. Observable objects of the exciton physics are few-particle states including a single hole and several quasielectrons. These two fields constitute a single whole with the analogous problems and approaches. One of these problems is the quasiparticle interaction law. It seems probable that exciton spectroscopy may become a very useful tool for investigating it. Indeed, the exciton spectroscopy deals with few-particle systems, has in its disposal the governing parameter $h / l$, and reach experimental techniques (selection rules, polarized spectra, etc.). Unification of the transport and spectroscopic techniques on the level of fundamental mechanisms seems highly promising.

\section{Acknowledgments}

I am grateful to A. L. Efros for numerous critical and suggestive discussions, to Y.-S. Wu for illuminating comments, to J. M. Worlock for critical reading the manuscript and valuable advice, to F. G. Pikus for advice related to computational procedures, and especially to V. M. Apalkov for a fruitful collaboration. The support by NSF Grant No. DMR-9116748 and San Diego Supercomputer Center where the computations were performed is acknowledged. 


\section{References}

1. D. C. Tsui, H. Stormer, and A. C. Gossard, Phys. Rev. Lett. 48, 1559 (1982).

2. R. B. Laughlin, Phys. Rev. Lett. 50, 13 (1983).

3. B. I. Halperin, Phys. Rev. Lett. 52, 1583 (1984).

4. F. G. M. Haldane, Phys. Rev. Lett. 67, 937 (1991).

5. S. M. Girvin, A. H. MacDonald, and P. M. Platzman, Phys. Rev. 33, 2481 (1986).

6. R. B. Laughlin, Physica 126B, 254 (1984).

7. D. Heiman, B. B. Goldberg, A. Pinczuk et al., Phys. Rev. Lett. 61, 605 (1988);

A. J. Turberfield, S. H. Heines, P. A. Wright et al., Phys. Rev. Lett. 65, 637 (1990);

E. M. Goldis, S. A. Brown, R. B. Dunford et al., Phys. Rev. 46, 7957 (1992);

D. Heiman, A. Pinczuk, M. Dahl et al., Surf. Sci. 305, 50 (1994).

8. A. Pinczuk, B. S. Dennis, L. N. Pfeiffer, and K. West, Phys. Rev. Lett. 70, 3983 (1993).

9. I. V. Kukushkin and V. B. Timofeev, JETP Lett. 44, 228 (1986).

10. V. M. Apalkov and E. I. Rashba, JETP Lett. 53, 442 (1991).

11. I. V. Kukushkin, N. J. Pulsford, K. von Klitzing et al., Surf. Sci. 263, 30 (1992);

I. V. Kukushkin, R. J. Haug, K. von Klitzing et al., Phys. Rev. Lett. 72, 736 (1994).

12. F. G. M. Haldane and E. H. Rezayi, Phys. Rev. Lett. 54, 259 (1985).

13. E. I. Rashba and V. M. Apalkov, in: Optical Phenomena in Semiconductor Structures of Reduced Dimensions, ed. by D. J. Lockwood and A. Pinczuk, Kluver, Dordrecht (The Netherlands, 1993), p. 63.

14. V. M. Apalkov and E. I. Rashba, Phys. Rev. B 46, 1628 (1992).

15. V. M. Apalkov and E. I. Rashba, Phys. Rev. Lett., submitted.

16. E. I. Rashba and M. E. Portnoi, JETP Lett. 57, 733 (1993), and unpublished paper for $q=3$.

17. E. I. Rashba and V. M. Apalkov, Proc. of the 11th Intern. Conf. "Applications of High Magnetic Fields in Semiconductor Physics", Cambridge, MA, 1994, in press.

18. V. M. Apalkov and E. I. Rashba, loc. cit. 